\title{
Peripheral zone volume ratio (PZ-ratio) is relevant with biopsy results and can increase the accuracy of current diagnostic modality
}

\author{
Yifan Chang ${ }^{1, *}$, Rui Chen ${ }^{1, *}$, Qingsong $\mathrm{Yang}^{2, *}, \mathrm{Xu} \mathrm{GaO}^{1}{ }^{1}$, Chuanliang $\mathrm{Xu}{ }^{1}$, Jianping \\ Lu$^{2}$, Yinghao Sun ${ }^{1}$ \\ ${ }^{1}$ Department of Urology, Changhai Hospital, Second Military Medical University, Shanghai 200433, China \\ ${ }^{2}$ Department of Radiology, Changhai Hospital, Second Military Medical University, Shanghai 200433, China \\ *These authors contributed equally to this work \\ Correspondence to: Yinghao Sun, email: sunyhsmmu@126.com \\ Jianping Lu, email: cjr.lujianping@vip.163.com
}

Keywords: biopsy, diagnosis, multiparametric MRI, peripheral zone volume ratio, prostate cancer

Received: June 13, $2016 \quad$ Accepted: March 21, $2017 \quad$ Published: March 31, 2017

Copyright: Chang et al. This is an open-access article distributed under the terms of the Creative Commons Attribution License (CC-BY), which permits unrestricted use, distribution, and reproduction in any medium, provided the original author and source are credited.

\section{ABSTRACT}

The current diagnostic modality of prostate cancer based on prostate specific antigen (PSA) and systematic biopsy is far from ideal in terms of over-diagnosing indolent prostate cancer and missing significant ones. Thus we integrated the peripheral zone volume ratio (PZ-ratio) for diagnostic refinement. This retrospective study included 247 consecutive patients who underwent initial transrectal ultrasoundguided systematic prostate biopsy from April 2014 to November 2015. Prostate volume was determined by semi-automatic contour on axial T2 weighted magnetic resonance imaging (MRI). PZ-ratio was inversely correlated with age $(r=-0.36$, $p<0.0001$ ). Adding PZ-ratio and MRI findings to the current predictive model (age, PSA density, percent-free PSA) significantly increased diagnostic accuracy in all patients (AUC: 0.871 vs. $0.812, p=0.0059$ ), but not in patient subgroup with PSA 4-10 $\mathrm{ng} / \mathrm{ml}$ (AUC: $0.863 \mathrm{vs} .0 .803, p=0.12$ ). The new model also significantly reduced the number of unnecessary biopsies while missing less significant cancers at a probability threshold of $\mathbf{2 5 \%}$. PZ-ratio is a potential tool in predicting biopsy results, and when added alone or in combination with MRI findings, the diagnostic accuracy can be further enhanced.

\section{INTRODUCTION}

The random nature of transrectal ultrasound (TRUS) guided systematic prostate biopsy sampling and low specificity of prostate specific antigen (PSA) have induced overtreatment of clinically indolent prostate cancer $(\mathrm{PCa})$ and missed diagnosis of significant ones [1]. Therefore, the American Urological Association (AUA) guidelines on PCa recommend against the use of PSA threshold alone as biopsy indicator [2]. Subsequently, PSA density (PSAD), PSA density of the transition zone (PSADTZ) [3] and other modified prostate volume (PV)-related parameters have been proposed and investigated [4-7]. However, the current use of the prostate ellipsoid formula in volume estimation has long been questioned, with a reported
10-20\% error compared with prostatectomy specimens [8-10]. Comparatively, PV estimation by contoured MR images is regarded as the most precise noninvasive method available [11].

Although the causal role between benign prostatic hyperplasia $(\mathrm{BPH})$ and $\mathrm{PCa}$ is yet to be clarified, enlargement of an aging prostate due to BPH is typically contributed by the transition zone (TZ), while peripheral zone (PZ) is typically considered as age-irrelevant [12]. Because larger glands are related to lower cancer detection rate [13-15], the ratio of zonal volume may have the potential in differentiating PCa patients from BPH individuals. Porcaro et al. [16] reported that the $\mathrm{PCa}$ risk in men with $\mathrm{TZ} / \mathrm{PZ}$ volume $\leq 1$ was 2.36 times greater than those $>1$. Qi et al. [17] proposed that a cut-off 
value of 0.47 in TZ index helped to identify unnecessary biopsies. Previous studies using the contoured method on MRI have observed significant correlation between central gland volume fraction and Gleason Score [18], and can better distinguish PCa patients [19]. However, these studies have a relatively small sample size with no further stratification of patients with PSA level of 4-10 ng/ml, and did not perform multivariate correlation analyses. Hence, its efficacy requires further research.

The purpose of the current study was to validate the diagnostic value of PZ-ratio (i.e., PZ volume divided by prostate volume) based upon contoured volume in axial T2 fat saturated MR images, and to evaluate whether incorporating this parameter provides any additional benefit in the current diagnostic modality in detecting clinically significant PCa.

\section{RESULTS}

\section{Patient characteristics}

A total of 247 consecutive patients with PSA $\geq$ $4.0 \mathrm{ng} / \mathrm{ml}$ or PSA $<4.0 \mathrm{ng} / \mathrm{ml}$ but with abnormal digital rectal examination (DRE) findings were included in the study, in which 88 cases (35.6\%) and 77 cases $(31.2 \%)$ were diagnosed with PCa and significant PCa, respectively. The median PSA level of PCa patients and biopsy-negative patients were 13.1 (interquartile range (IQR), 8.28 to 21.8 ) $\mathrm{ng} / \mathrm{mL}$ and 8.6 (IQR, 6.18 to 13.0$) \mathrm{ng} / \mathrm{ml}$, respectively. The median age at biopsy was 68 years in PCa patients and 64 in biopsy-negative patients. The median PZ-ratio was 38.2\% (IQR, 28.8\% to $47.6 \%$ ) and $27.7 \%$ (IQR, $19.78 \%$ to $38.8 \%$ ) in $\mathrm{PCa}$ patients and biopsy-negative patients, respectively $(p=0.0001)$ (Figure 1A). Clinical stage and Gleason Score after biopsy was listed in Table 1. BPH was histologically confirmed in 8 of $88 \mathrm{PCa}$ patients $(9.1 \%)$ and 89 of 159 biopsy-negative patients $(56.0 \%)(p<0.0001)$, and 4 of 28 $(14.3 \%)$ and 38 of $80(47.5 \%)$ in PCa patients and biopsynegative patients with PSA of 4-10 ng/ml, respectively $(p<0.0001)$.

\section{Association between PZ-ratio and other clinical parameters}

PZ-ratio was correlated with PV $(r=-0.5698$, $p<0.0001)$, percent-free PSA (\%fPSA) $(r=-0.326$, $p<0.0001)$ and age $(r=-0.36, p<0.0001)$ (Figure $2 \mathrm{E}$ ) but not with PSA level $(p=0.206)$ or PSAD $(p=0.107)$. Older men were more likely to have lower PZ-ratio (Figure 1E). Since age would significantly influence clinical decisionmaking, we tested whether the relationship between PZratio and biopsy results varied in patients in different age ranges. PZ-ratio could differentiate PCa from biopsynegative patients aged 60-69 years and over 69 years ( $p<0.0001, p=0.0024$, respectively) but failed to differentiate prostate cancer from biopsy-negative ones in those younger than 59 years $(p=0.691)$ (Figure $1 F)$.

\section{Correlation of PZ-ratio and biopsy results}

PZ-ratio was effective in predicting $\mathrm{PCa}$ in all patients and patients with PSA 4-10 ng/ml. The predicted probability of prostate cancer in all patients (Supplementary Figure 2A) and patients with PSA 4-10 ng/ml (Supplementary Figure 2B) increased with the value of PZratio. The AUC of PZ-ratio was 0.672 (95\% CI, 0.603 to $0.741)$ and 0.659 (95\% CI, 0.588 to 0.730$)$ in predicting $\mathrm{PCa}$ and significant PCa (Table 2). For patients with PSA of 4-10 ng/ml, the AUC of PZ-ratio was 0.676 (95\% CI, 0.557 to 0.795$)$ and 0.642 (95\% CI, 0.498 to 0.786$)$ in predicting $\mathrm{PCa}$ and significant $\mathrm{PCa}$, respectively. The sensitivity and specificity of PZ-ratio in predicting biopsy result was summarized (Supplementary Table 2). PZ-ratio cutoff value of $34.9 \%$ could obtain sensitivity of $67.9 \%$ and specificity of $67.5 \%$ in predicting significant $\mathrm{PCa}$ in patients with PSA of 4-10 ng/ml. To ensure the detection of $90 \%$ significant PCa, the PZ-ratio cutoff value should be $20.9 \%$. At a cutoff value of $47.8 \%$, the positive predicted value of PZ-ratio was 0.5. The AUC of PZ-ratio was not higher than traditional predictors, thus we further evaluated if adding PZ-ratio into the diagnostic model would benefit the current clinical practice.

\section{Logistic regression analysis}

In univariate logistic regression models, PZ-ratio was associated with biopsy result ( $\mathrm{PCa}$ or non-PCa, and significant $\mathrm{PCa}$ or insignificant $\mathrm{PCa}$ and benign tissue) in all patients (Supplementary Table 3). While PZ-ratio was only significantly correlated with $\mathrm{PCa}$ but only on a borderline significant level with significant PCa $(p=0.105)$, further multivariable logistic regression models including base model (age, PSAD, \%fPSA) and base model + PZ-ratio, base model + MRI, base model + PZ-ratio + MRI for PCa and significant PCa, respectively, were listed in Supplementary Table 4.

\section{Diagnostic accuracy of diagnostic models with PZ-ratio and MRI findings}

Adding PZ-ratio into the base model would lead to improvement of AUC in all patients and in patients with PSA 4-10 ng/ml. In the prediction of all PCa in all patients, the AUC of base model, base model + PZ-ratio, base model + MRI, base model + MRI + PZ-ratio was 0.812, 0.839, 0.854 , and 0.871 , respectively (Table 2, Supplementary Figure 3A). The improvement of AUC was 0.059 $(p=0.0059)$ between base model + MRI + PZ-ratio and base model. Similarly, the AUC of base model + MRI + PZ-ratio was 0.890 in predicting significant PCa, significantly higher than that of the base model $(0.890$ 
Table 1: Clinical characteristics of men with positive and negative biopsy

\begin{tabular}{|c|c|c|c|c|c|c|}
\hline & \multicolumn{3}{|c|}{ All patients $(n=247)$} & \multicolumn{3}{|c|}{ PSA 4.0-10.0 $\mathrm{ng} / \mathrm{ml}(n=108)$} \\
\hline & Positive & Negative & $P$ value & Positive & Negative & $P$ value \\
\hline No. of pts, \% & $88(35.6)$ & $159(64.4)$ & & $28(25.9)$ & $80(74.1)$ & \\
\hline $\begin{array}{l}\text { Clinically significant Ca } \\
\text { Age, median (IQR), years }\end{array}$ & $\begin{array}{c}77(31.2) \\
68(61,76)\end{array}$ & $\begin{array}{l}170(68.8) \\
64(59,71)\end{array}$ & $P^{*}=0.0007$ & $\begin{array}{c}19(17.6) \\
66(61,77)\end{array}$ & $\begin{array}{c}89(82.4) \\
62(57,68)\end{array}$ & $P^{*}=0.0176$ \\
\hline PSA, median (IQR), ng/ml & $13.1(8.2,21.8)$ & $8.6(6.1,13.0)$ & $P^{*}<0.0001$ & $7.6(6.4,8.5)$ & $7.0(6.0,8.5)$ & $P^{*}=0.3094$ \\
\hline \multicolumn{7}{|l|}{ Clinical stage, $\%$} \\
\hline T1c & $10(11.4)$ & & & $4(14.3)$ & & \\
\hline T2a/T2b & $53(60.2)$ & & & $16(57.1)$ & & \\
\hline T2c & $24(27.3)$ & & & $8(28.6)$ & & \\
\hline T3 and above & $1(1.1)$ & & & $0(0.0)$ & & \\
\hline \multicolumn{7}{|l|}{ Gleason Score, \% } \\
\hline$<7$ & $22(25.0)$ & & & $11(39.3)$ & & \\
\hline$=7$ & $28(31.8)$ & & & $9(32.1)$ & & \\
\hline$>7$ & $27(30.7)$ & & & $5(17.9)$ & & \\
\hline Missing data & $11(12.5)$ & & & $3(10.7)$ & & \\
\hline Prostate volume, $\mathrm{ml}$ & $39.9(30.7,56.9)$ & $56.6(41.0,75.5)$ & $P^{*}<0.0001$ & $39.3(29.6,53.9)$ & $54.5(43.2,69.9)$ & $P^{*}=0.0007$ \\
\hline$\%$ fPSA, $\%$ & $11.3(8.2,15.7)$ & $15.3(11.1,21.5)$ & $P^{*}<0.0001$ & $11.9(7.2,17.4)$ & $15.3(11.3,20.8)$ & $P^{*}=0.0099$ \\
\hline PSAD & $0.32(0.18,0.54)$ & $0.15(0.10,0.24)$ & $P^{*}<0.0001$ & $0.18(0.14,0.23)$ & $0.14(0.09,0.17)$ & $P^{*}=0.0003$ \\
\hline PZ-ratio, \% & $38.2(28.8,47.6)$ & $27.7(19.7,38.8)$ & $P^{\#}=0.0001$ & $39.9(32.3,48.3)$ & $30.5(20.4,39.5)$ & $P^{\#}=0.0196$ \\
\hline Positive MRI, \% & 78/88 (88.6) & $59 / 159(37.1)$ & $P^{* *}<0.0001$ & $24 / 28(85.7)$ & $29 / 80(36.3)$ & $P^{* *}<0.0001$ \\
\hline BPH rate, \% & $8 / 88(9.1)$ & $89 / 159(56.0)$ & $P^{* *}<0.0001$ & $4 / 28(14.3)$ & $38 / 80(47.5)$ & $P^{* *}<0.0001$ \\
\hline
\end{tabular}

IQR: interquartile range; No. of pts: number of patients; PSA, prostate-specific antigen; \%fPSA, percent free PSA; PSAD, prostate-specific antigen density; PZ-ratio: peripheral zone ratio; MRI: magnetic resonance imaging; BPH, benign prostatic hyperplasia. $P^{*}$ : Mann-Whitney $U$ test; $P^{*}$ : independent sample test; $P^{* *}$ : Chi-square test.

vs. 0.843, $p=0.0227$ ) (Supplementary Figure 3C). However, in patients with PSA 4-10 ng/ml, although the improvement in AUC was similar, the result was not statistically significant in predicting PCa (0.863 vs. 0.803 , $p=0.125$ ) (Supplementary Figure $3 \mathrm{~B}$ ) or significant $\mathrm{PCa}$ (0.899 vs. $0.846, p=0.176)$ (Supplementary Figure 3D).

\section{Decision curve analysis for predicting significant PCa}

As the decision curve indicated, the base model + MRI + PZ-ratio was superior to the base model in the defined range of interest (15-40\% probability) in predicting significant $\mathrm{PCa}$ in all patients (Figure 2A) and patients with PSA 4-10 ng/ml (Figure 2B) with a higher net benefit (Supplementary Table 5). Both models outperform the "treat all" strategy of performing biopsies on every patient. Supplementary Table 6 shows the number of significant PCa missed and the reduction in biopsies according to threshold probability for base model and base model + MRI + PZ-ratio. For instance, if a probability threshold of $25 \%$ was used in all patients, $3(3.8 \%)$ significant $\mathrm{PCa}$ would be missed while avoiding 73 (43.2\%) biopsies using the base model; adopting the base model + MRI + PZratio would save $98(58.1 \%)$ of unnecessary biopsies at the cost of missing $4(5.1 \%)$ significant prostate cancers. In patients with PSA 4-10 ng/ml, the base model + MRI + PZratio would save more unnecessary biopsies (64 (71.9\%) vs. $59(66.3 \%))$ while missing less significant cancers $(1(5.3 \%)$ vs. $2(10.5 \%))$ at a probability threshold of $25 \%$ for intervention.

\section{DISCUSSION}

Although the etiology of $\mathrm{BPH}$ and $\mathrm{PCa}$ and their possible correlation is not clearly illustrated [25], epidemiologically, most biopsy candidates are in their $50 \mathrm{~s}$ to $70 \mathrm{~s}$, in which case the incidence of BPH dramatically increases [26]. This contributes, at various extents, to the enlargement of the prostate, and almost exclusively, of TZ [27]. Thus, one may hypothesize that the elevation of PSA in biopsynegative cases is likely to be contributed by $\mathrm{BPH}$ in otherwise healthy males [28]. As a result, PZ-ratio may serve as a volumetric parameter that has the potential to differentiate PCa patients from non-cancer ones, and to provide an additional tool in determining biopsy candidates. In this study, PZ-ratio was able to differentiate $\mathrm{PCa}$ patients from $\mathrm{BPH}$ men in ages above 60 years, but results were not statistically different in patients under 60 years. Firstly, men aged 50 to 60 may present, if any, only mild BPH. Thus it is reasonable that 
PZ-ratio in this subgroup may not be markedly different between these two populations. Secondly, the sample size of this younger-aged subgroup may not be sufficient.

When used alone, PZ-ratio was not a good predictor in all patients (AUC $=0.672$ ) and in PSA 4-10 ng/ml subgroup $(A U C=0.676)$. However, when added to the base model, PZ-ratio alone or in combination with MRI findings were both able to further improve the AUC to up to 0.899 . For patients with PSA 4-10 ng/ml, the improvement was not statistically significant. For one, the AUC of all predictors were lower in patients with PSA 4-10 ng/ml than in the overall patients (Table 2); also, because the difference of PZ-ratio between PCa and noncancer patients in this subgroup was relatively smaller than in the overall population; or, it may result from insufficient sample size; nevertheless, decision curve analysis indicated
A

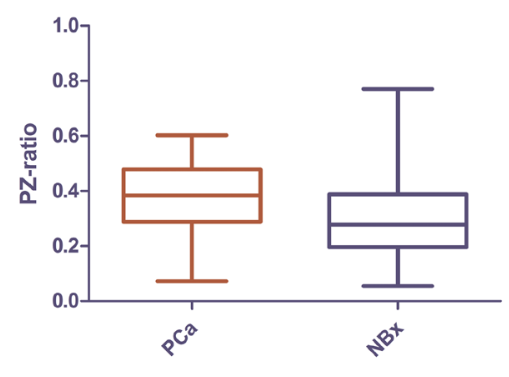

D

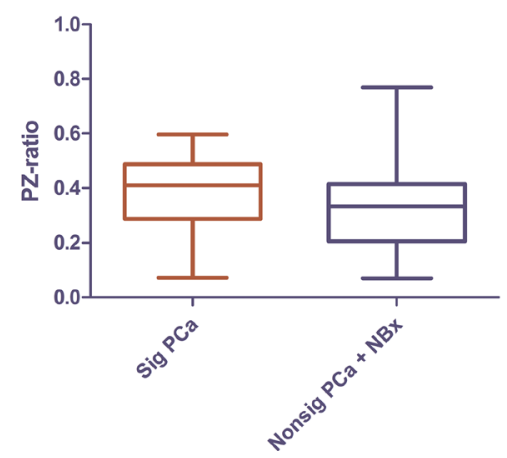

B

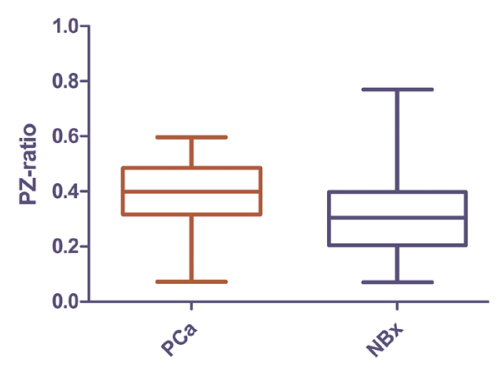

E

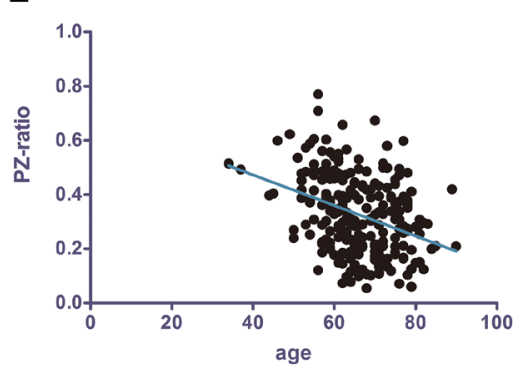

C
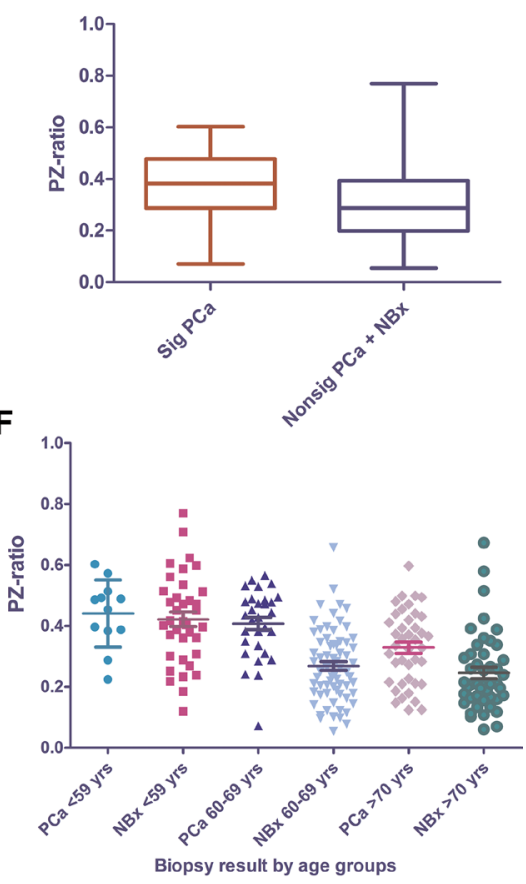

Figure 1: PZ-ratio in PCa and biopsy-negative patients in all PCa of all patient group (A) and of patients with PSA of 4-10 ng/ml (B)significant PCa vs. insignificant PCa + negative biopsy of all patient group (C) and patients with PSA of 4-10 ng/ml (D) and the relevance of PZ-ratio with age in all ages $(\mathbf{E})$ and subdivided by age groups $(\mathbf{F})$. PZ-ratio, peripheral zone volume ratio; PCa, prostate cancer; NBx, negative biopsy; Sig PCa, significant prostate cancer; nonsig PCa, non-significant prostate cancer.

A

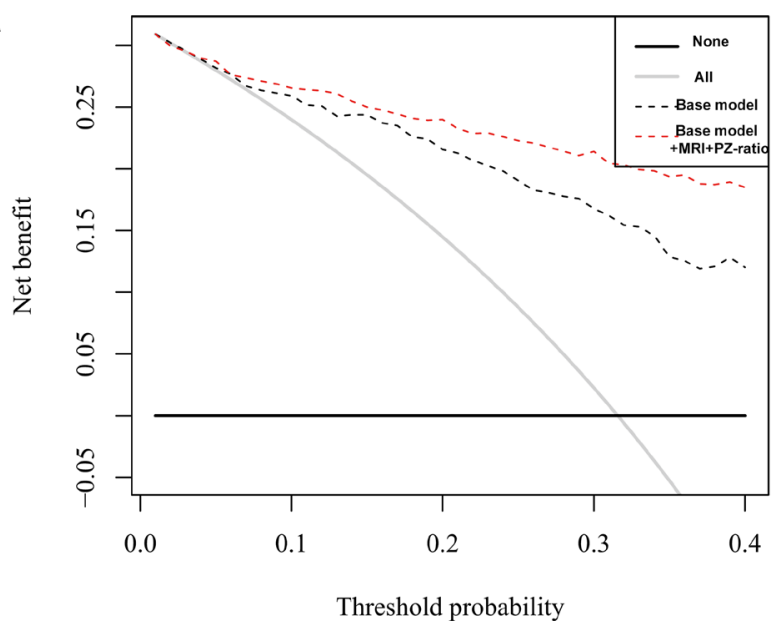

B

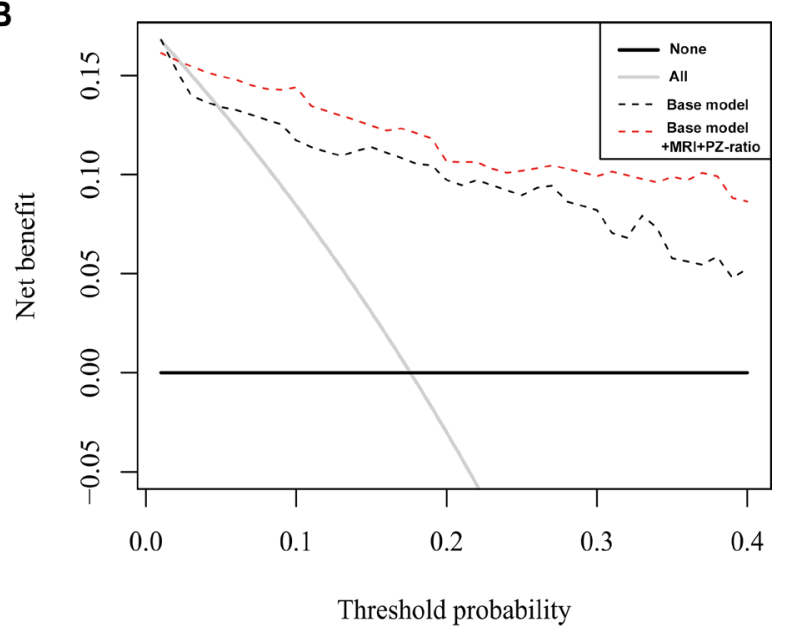

Figure 2: Decision curve analysis of predicting significant PCa with base model vs. base model + MRI + PZ-ratio in all PSA group (A) and patients with PSA of 4-10 $\mathrm{ng} / \mathrm{ml}$ (B). 
Table 2: Diagnostic accuracy of predictors and prediction models in predicting prostate cancer or significant prostate cancer

\begin{tabular}{|c|c|c|c|c|c|}
\hline & \multirow{2}{*}{ Predictors } & \multicolumn{2}{|c|}{ Predicting PCa } & \multicolumn{2}{|c|}{ Predicting Significant PCa } \\
\hline & & AUC & $95 \% \mathrm{CI}$ & AUC & $95 \% \mathrm{CI}$ \\
\hline \multirow{9}{*}{ All Pts $(n=247)$} & tPSA & 0.679 & $0.609-0.749$ & 0.722 & $0.652-0.792$ \\
\hline & PZ-ratio & 0.672 & $0.603-0.741$ & 0.659 & $0.588-0.730$ \\
\hline & MRI & 0.758 & $0.707-0.808$ & 0.760 & $0.711-0.809$ \\
\hline & PSAD & 0.757 & $0.694-0.820$ & 0.781 & $0.717-0.844$ \\
\hline & $\%$ fPSA & 0.657 & $0.588-0.726$ & 0.653 & $0.583-0.724$ \\
\hline & Model & 0.812 & $0.758-0.866$ & 0.843 & $0.792-0.893$ \\
\hline & Model+PZ-ratio & 0.839 & $0.787-0.891$ & 0.869 & $0.822-0.917$ \\
\hline & Model+MRI & 0.854 & $0.807-0.900$ & 0.874 & $0.830-0.919$ \\
\hline & Model+MRI+PZ-ratio & 0.871 & $0.825-0.917$ & 0.890 & $0.845-0.934$ \\
\hline \multirow{9}{*}{$\begin{array}{l}\text { Pts PSA 4-10 ng/ml } \\
(n=108)\end{array}$} & tPSA & 0.565 & $0.441-0.688$ & 0.522 & $0.389-0.655$ \\
\hline & PZ-ratio & 0.676 & $0.557-0.795$ & 0.642 & $0.498-0.786$ \\
\hline & MRI & 0.747 & $0.663-0.832$ & 0.777 & $0.704-0.850$ \\
\hline & PSAD & 0.729 & $0.620-0.839$ & 0.692 & $0.555-0.830$ \\
\hline & $\% \mathrm{fPSA}$ & 0.664 & $0.550-0.779$ & 0.632 & $0.498-0.766$ \\
\hline & Model & 0.803 & $0.693-0.913$ & 0.846 & $0.728-0.964$ \\
\hline & Model+PZ-ratio & 0.851 & $0.766-0.936$ & 0.862 & $0.747-0.976$ \\
\hline & Model+MRI & 0.822 & $0.715-0.929$ & 0.888 & $0.792-0.984$ \\
\hline & Model+MRI+PZ-ratio & 0.863 & $0.776-0.951$ & 0.899 & $0.795-1.000$ \\
\hline
\end{tabular}

PCa: prostate cancer; PSA: prostate-specific antigen; PZ-ratio: peripheral zone ratio; MRI: magnetic resonance imaging; OR: odds ratio; CI: confidence interval; AUC: area under the curve.

that the model with PZ-ratio and MRI-findings was able to significantly reduce the number of unnecessary biopsies at the cost of similar or less significant PCa missed in all patients and patients with PSA 4-10 ng/ml.

We believe several aspects of this study strengthened its reliability. Firstly, application of the MRI-based contoured method offers a more precise estimation of prostate volume. Apart from inherent limitations of the ellipsoid formula under TRUS in determining PV, which may underestimate PV by at least $10 \%$, [8] the reproducibility of TRUS is also limited due to interobserver variability. Comparatively, MRI contour is proven to be more precise ( $r=0.93$ vs. 0.81$)$ than TRUS, $[11,29]$ and also an easy and simple method with high reproducibility for radiologists and urologists to master after simple training. Our preference of fat saturated T2WI images is explained by the fact that PZ appears hyperintense and more discernable from $\mathrm{TZ}$ and other periprostatic tissue [12]. Secondly, it is noted that central zone appears as bilateral basal hypointense zones, or, 'moustache sign' on T2 fat saturated images. It is possible that the central zone is mistreated as suspected lesions in the PZ [30], or simply being included as part of PZ, causing additional measurement error. These special attentions may help to achieve more accurate MRI interpretation and more precise measurement results. Thirdly, although this is not the first study focusing on MRI-based PZ-ratio in the diagnosis of $\mathrm{PCa}$, it is, to our knowledge, the first study to test PZ-ratio in a real clinical scenario. It is also the first time that MRI-based PZ-ratio was evaluated in improving current clinical practice and prediction models.

Several limitations of this study should be noted. Firstly, systematic biopsy was performed by three attending urologists in our study, and sampling error may occur. Secondly, the sample size in patients with PSA 4-10 $\mathrm{ng} / \mathrm{ml}$ was rather inadequate, and further investigations with particular attention to this subgroup is needed. Thirdly, we failed to discriminate TZ PCa from PZ ones. TZ cancers occur far less likely than PZ ones, and are typically not detected by systematic biopsy, because $\mathrm{TZ}$ is usually not sampled. Fourthly, since this study was conducted in Chinese population, it concerns us whether the racial difference on PV would influence the efficacy 
of PZ-ratio. Further multiethnic investigations in western population is needed. Also, the retrospective and singlecentered nature of this study has its inherent limitations.

\section{MATERIALS AND METHODS}

\section{Study population}

This study included urology outpatients with PSA levels over $4.0 \mathrm{ng} / \mathrm{ml}$ or PSA $<4.0 \mathrm{ng} / \mathrm{ml}$ but with suspicious DRE findings. Between April 2014 and December 2015, a total of 247 consecutive patients receiving multiparametric MRI (mpMRI) before 12-core systematic TRUS-guided transrectal biopsy in Changhai Hospital, Shanghai, China, were involved in this study. Ethical approval was acquired from local institutional review board, and informed consent was signed by patients before the study.

\section{Image technique and biopsy scheme}

MR imaging was performed with a 3.0T MR scanner (Magnetom Skyra, Siemens Medical Solutions, Erlangen, Germany). The prostate MRI protocols included T1WI TSE, triplanar (axial, sagittal and coronal) T2WI TSE, diffusion weighted imaging (DWI) and dynamic contrast-enhanced imaging (DCE) by using an 18-channel phased-array coil. Imaging parameters were detailed in Supplementary Table 1. The interpretation of MRI findings was based on PIRADS V2 results performed by an attending radiologist (Q.Y.) with 10 years of experience in prostate MRI. Prostate biopsies were performed by three attending urologists. Additional biopsy cores were taken on suspected spots according to mpMRI findings (i.e., cognitive fusion).

\section{Pathological review}

Two genitourinary pathologists with 21 and 7 years' experience in prostate histology reviewed all histopathologic slides from prostate biopsy. Clinically significant prostate cancer was defined according to D'Amico's Criteria (i.e., either PSA level $>10 \mathrm{ng} / \mathrm{ml}$ or Gleason pattern 4 or 5 or clinical stage over T2a) [20].

\section{Measurement of prostate volume and peripheral zone volume}

PV and PZ were semi-automatically segmented on axial fat-saturated T2WI MR images, in OsiriX v5.8.1 (Pixmeo, Geneva, Switzerland), as reported previously $[21,22]$. The radiologist (Q.Y.) with 10 years of experience in prostate MRI reviewed and manually corrected the contoured areas, under the supervision of a senior radiologist (J.L.) with over 30 years' experience on prostate imaging. PV and PZ were calculated by multiplying the sum of contoured area on each slices by slice thickness (Supplementary Figure 1).

\section{Statistical analysis}

Linear correlation analyses were performed to evaluate the association between PZ-ratio and other clinical parameters (age, PSA, \%fPSA, PV and PSAD). The predicted probability of biopsy-detected PCa for a given PZ-ratio value was calculated using locally weighted scatterplot ("lowess") smoothing [23]. Univariate logistic regression analyses were performed to explore the relationship between $\mathrm{PZ}$ volume and PZratio with biopsy result (PCa or non-PCa, and significant $\mathrm{PCa}$ or insignificant $\mathrm{PCa}$ and benign tissue). Multivariate logistic regression analyses were performed to explore if PZ-ratio is an independent predictor of biopsy result. We evaluated the diagnostic accuracy of a base model constructed with clinical predictors (age, PSAD, \%fPSA). We also evaluated the diagnostic accuracy of the logistic prediction model with PZ-ratio (base model + PZ-ratio), with MRI results (base model $+\mathrm{MRI}$ ), and the model with PZ-ratio and MRI results (base model + PZ-ratio + MRI). The predictive accuracy of PZ-ratio, other predictors and prediction models was assessed by area under the curve (AUC) of receiver operating characteristic (ROC) curve. Sensitivity, specificity, positive and negative predicted values were calculated. The comparison of AUC was calculated by $z$ test. In addition, decision curve analysis described by Vickers et al. [24] was performed to assess the clinical utility of prediction models with/without PZratio and MRI findings by quantifying the net benefits at a spectrum of threshold probabilities. In this case, we focused on $15-40 \%$, in which clinical decision making is particularly difficult. All $p$ values were two-sided and $p<0.05$ was considered statistically significant. All statistical analyses were performed using MedCalc v.10.4.7.0 (MedCalc Software bvba, Mariakerke, Belgium) and $\mathrm{R}$ version 3.1.3 (R foundation for Statistical Computing, Vienna, Austria, http://www.R-project.org) with the Design and Hmisc libraries added.

\section{CONCLUSIONS}

PZ-ratio has the potential as predictor for biopsy results. PZ-ratio is inversely proportionate with age, and can discriminate prostate cancer patients from biopsy-negative ones in men above 60. Alone or in combination with MRI findings, it may offer clinicians additional value based on the current diagnostic modality. Further prospective studies are required to validate its consideration for wide application.

\section{CONFLICTS OF INTEREST}

This study received institutional review board approval. The authors declare no financial interests and/ or other relationships. 


\section{REFERENCES}

1. Bjurlin MA, Meng X, Le Nobin J, Wysock JS, Lepor H, Rosenkrantz AB, Taneja SS. Optimization of prostate biopsy: the role of magnetic resonance imaging targeted biopsy in detection, localization and risk assessment. J Urol. 2014; 192:648-58.

2. Greene KL, Albertsen PC, Babaian RJ, Carter HB, Gann PH, Han M, Kuban DA, Sartor AO, Stanford JL, Zietman A, Carroll P. Prostate specific antigen best practice statement: 2009 update. J Urol. 2013; 189: S2-s11.

3. Kalish J, Cooner WH, Graham SD Jr. Serum PSA adjusted for volume of transition zone (PSAT) is more accurate than PSA adjusted for total gland volume (PSAD) in detecting adenocarcinoma of the prostate. Urology. 1994; 43:601-6.

4. Benson MC, Whang IS, Olsson CA, McMahon DJ, Cooner WH. The use of prostate specific antigen density to enhance the predictive value of intermediate levels of serum prostate specific antigen. J Urol. 1992; 147:817-21.

5. Nowroozi M, Ayati M, Jamshidian H, Arbab A, Ghorbani H, Amini E, Hakima H, Salehi S, Ghadian A. Transition zone prostate specific antigen density improves prostate cancer detection in Iranian men. Nephrourol Mon. 2015; 7: e26752.

6. Koo KC, Lee DH, Lee SH, Chung BH. Peripheral zone prostate-specific antigen density: an effective parameter for prostate cancer prediction in men receiving 5alphareductase inhibitors. Prostate Int. 2013; 1:102-8.

7. Kosaka T, Mizuno R, Shinojima T, Miyajima A, Kikuchi E, Tanaka N, Shinoda K, Morita S, Mikami S, Oya M. The implications of prostate-specific antigen density to predict clinically significant prostate cancer in men $</=50$ years. Am J Clin Exp Urol. 2014; 2:332-6.

8. Partin AW, Oesterling JE. The clinical usefulness of prostate specific antigen: update 1994. J Urol. 1994; 152:1358-68.

9. Zlotta AR, Djavan B, Marberger M, Schulman CC. Prostate specific antigen density of the transition zone: a new effective parameter for prostate cancer prediction. J Urol. 1997; 157:1315-21.

10. Hricak H, Jeffrey RB, Dooms GC, Tanagho EA. Evaluation of prostate size: a comparison of ultrasound and magnetic resonance imaging. Urol Radiol. 1987; 9:1-8.

11. Rahmouni A, Yang A, Tempany CM, Frenkel T, Epstein J, Walsh P, Leichner PK, Ricci C, Zerhouni E. Accuracy of invivo assessment of prostatic volume by MRI and transrectal ultrasonography. J Comput Assist Tomogr. 1992; 16:935-40.

12. Turkbey B, Huang R, Vourganti S, Trivedi H, Bernardo M, Yan P, Benjamin C, Pinto PA, Choyke PL. Age-related changes in prostate zonal volumes as measured by highresolution magnetic resonance imaging (MRI): a crosssectional study in over 500 patients. BJU Int. 2012; 110:1642-7.

13. Chen ME, Troncoso P, Johnston D, Tang K, Babaian RJ. Prostate cancer detection: relationship to prostate size. Urology. 1999; 53:764-8.
14. Ankerst DP, Till C, Boeck A, Goodman P, Tangen CM, Feng Z, Partin AW, Chan DW, Sokoll L, Kagan J, Wei JT, Thompson IM. The impact of prostate volume, number of biopsy cores and American Urological Association symptom score on the sensitivity of cancer detection using the Prostate Cancer Prevention Trial risk calculator. J Urol. 2013; 190:70-6.

15. Wu YS, Na R, Xu JF, Bai PD, Jiang HW, Ding Q. The influence of prostate volume on cancer detection in the Chinese population. Asian J Androl. 2014; 16:482-6.

16. Porcaro AB, Novella G, Molinari A, Terrin A, Minja A, De Marco V, Martignoni G, Brunelli M, Cerruto MA, Curti P, Cavalleri S, Artibani W. Prostate volume index and chronic inflammation of the prostate type IV with respect to the risk of prostate cancer. Urol Int. 2015; 94:270-85.

17. Qi TY, Chen YQ, Jiang J, Zhu YK, Yao XH, Wang XJ. Utility of the transition zone index for identification of prostate cancer in Chinese men with intermediate PSA levels. Int Urol Nephrol. 2012; 44:807-15.

18. Karademir I, Shen D, Peng Y, Liao S, Jiang Y, Yousuf A, Karczmar G, Sammet S, Wang S, Medved M, Antic T, Eggener S, Oto A. Prostate volumes derived from MRI and volume-adjusted serum prostate-specific antigen: correlation with Gleason score of prostate cancer. AJR Am J Roentgenol. 2013; 201:1041-8.

19. Peng Y, Shen D, Liao S, Turkbey B, Rais-Bahrami S, Wood B, Karademir I, Antic T, Yousef A, Jiang Y, Pinto PA, Choyke PL, Oto A. MRI-based prostate volume-adjusted prostate-specific antigen in the diagnosis of prostate cancer. J Magn Reson Imaging. 2015; 42:1733-9.

20. Bastian PJ, Carter BH, Bjartell A, Seitz M, Stanislaus P, Montorsi F, Stief CG, Schröder F. Insignificant prostate cancer and active surveillance: from definition to clinical implications. Eur Urol. 2009; 55:1321-30.

21. Blackledge MD, Collins DJ, Koh DM, Leach MO. Rapid development of image analysis research tools: Bridging the gap between researcher and clinician with pyOsiriX. Comput Biol Med. 2016; 69:203-12.

22. Dianat SS, Rancier Ruiz RM, Bonekamp D, Carter HB, Macura KJ. Prostate volumetric assessment by magnetic resonance imaging and transrectal ultrasound: impact of variation in calculated prostate-specific antigen density on patient eligibility for active surveillance program. J Comput Assist Tomogr. 2013; 37:589-95.

23. Cleveland WS. Robust Locally Weighted Regression and Smoothing Scatterplots. J Am Stat Assoc. 1979; 74: 829-36.

24. Vickers AJ, Elkin EB. Decision curve analysis: a novel method for evaluating prediction models. Med Decis Making. 2006; 26:565-74.

25. Alcaraz A, Hammerer P, Tubaro A, Schroder FH, Castro R. Is there evidence of a relationship between benign prostatic hyperplasia and prostate cancer? Findings of a literature review. Eur Urol. 2009; 55:864-73.

26. McVary KT. BPH: epidemiology and comorbidities. Am J Manag Care. 2006; 12:S122-8. 
27. Garvey B, Turkbey B, Truong H, Bernardo M, Periaswamy S, Choyke PL. Clinical value of prostate segmentation and volume determination on MRI in benign prostatic hyperplasia. Diagn Interv Radiol. 2014; 20:229-33.

28. De Nunzio C, Kramer G, Marberger M, Montironi R, Nelson W, Schröder F, Sciarra A, Tubaro A. The controversial relationship between benign prostatic hyperplasia and prostate cancer: the role of inflammation. Eur Urol. 2011; 60:106-17.
29. Nathan MS, Seenivasagam K, Mei Q, Wickham JE, Miller RA. Transrectal ultrasonography: why are estimates of prostate volume and dimension so inaccurate? Br J Urol. 1996; 77:401-7.

30. Panebianco V, Barchetti F, Barentsz J, Ciardi A, Cornud F, Futterer J, Villeirs G. Pitfalls in Interpreting mp-MRI of the Prostate: A Pictorial Review with Pathologic Correlation. Insights Imaging. 2015; 6:611-30. 